Received: 5 March 2019

Accepted: 31 May 2019

Published online: 17 June 2019

\section{Contribution of DNA methylation to the expression of FCGRT in human liver and myocardium}

\author{
R. B. Cejas ${ }^{1}$, D. C. Ferguson ${ }^{1}$, A. Quiñones-Lombraña $\mathbb{D}^{1}$, J. E. Bard $\mathbb{D}^{2}$ \& J. G. Blanco ${ }^{1}$
}

FcRn mediates recycling and transcytosis of IgG and albumin in various cell types. The MHC-classI-like protein of the FCRn heterodimer is encoded by FCGRT. Few determinants of variable FCGRT expression in humans have been identified so far. In this study, we investigated the presence of DNA methylation in regulatory regions of FCGRT in samples of human liver and myocardium tissue, and we examined the impact of FCGRT methylation on FCRn expression in model cell lines. Quantitative DNA methylation analysis of the FCGRT locus revealed differentially methylated regions in DNA from liver and myocardium. Methylation status in individual CpG sites correlated with FCGRT mRNA expression. Data from model cell lines suggest that differential methylation in the -1058 to -587 bp regulatory region of FCGRT contributes to FcRn expression. Chromatin immunoprecipitation assays indicate that CpG site methylation impacts the binding of the methylation sensitive transcription factors Zbtb7a and $\mathrm{Sp1}$. This study provides a foundation to further define the contribution of epigenetic factors during the control of FcRn expression and IgG traffic in human tissues.

The human neonatal Fc receptor $(\mathrm{FcRn})$ is a heterodimer comprised of a MHC-class-I-like heavy chain and beta-2-microglobulin ${ }^{1}$. The MHC-class-I-like protein of FcRn is encoded by the FCGRT gene. FcRn mediates recycling and transcytosis of IgG and albumin in various cell types ${ }^{2-5}$. In humans, FcRn is the only high affinity and $\mathrm{pH}$ specific receptor of IgG $\mathrm{G}^{6}$. In liver, FcRn contributes to maintain homeostatic concentrations of albumin and IgG by preventing their catabolism 7 . FcRn-mediated recycling allows IgG and albumin to have half-lives in blood approaching three weeks ${ }^{8,9}$. FcRn is also an important determinant for the pharmacokinetic and pharmacodynamic properties of protein therapeutics that contain Fc domains such as monoclonal antibody drugs ${ }^{10,11}$.

Variable FCGRT gene expression may contribute to drive the expression of the FcRn heterodimer under normal and pathological conditions ${ }^{12-14}$. Few determinants of variable FCGRT expression in humans have been identified so far. For example, Liu et al. have shown that FCGRT expression is decreased by INF-gamma via the JAK/STAT pathway and increased by proinflammatory stimulus through intronic NF-kappaB elements ${ }^{15,16}$. Polymorphic variable number of tandem repeats (VNTR) in the promoter region impact FcRn expression ${ }^{17}$. A previous report from our group described $h s a-m i R-3181$ as a potential regulator of FCGRT expression ${ }^{18}$.

DNA methylation contributes to the control of gene expression through processes involving modifications of chromatin structure that impact protein-DNA interactions ${ }^{19}$. In general, DNA methylation is associated with gene repression, although the resulting effect on gene expression is also dependent upon the location of differentially methylated regions ${ }^{20,21}$. DNA methylation occurs predominantly at CpG dinucleotides, and CpG density and methylation status on regulatory regions may affect gene expression ${ }^{22}$. The extent of FCGRT methylation and its potential contribution to differential FcRn expression remains to be defined. In this study, we investigated the presence of DNA methylation in regulatory regions of FCGRT in samples of human liver and myocardial tissue. We examined the impact of FCGRT methylation on the expression of FcRn in model cell lines. Our results provide insights into the role of variable DNA methylation in FCGRT during the expression of FcRn.
${ }^{1}$ Department of Pharmaceutical Sciences, School of Pharmacy and Pharmaceutical Sciences, The State University of New York at Buffalo, Buffalo, NY, 14214, USA. ${ }^{2}$ Genomics and Bioinformatics Core, New York State Center of Excellence in Bioinformatics and Life Sciences, The State University of New York at Buffalo, Buffalo, NY, 14203, USA. Correspondence and requests for materials should be addressed to J.G.B. (email: jgblanco@buffalo.edu) 

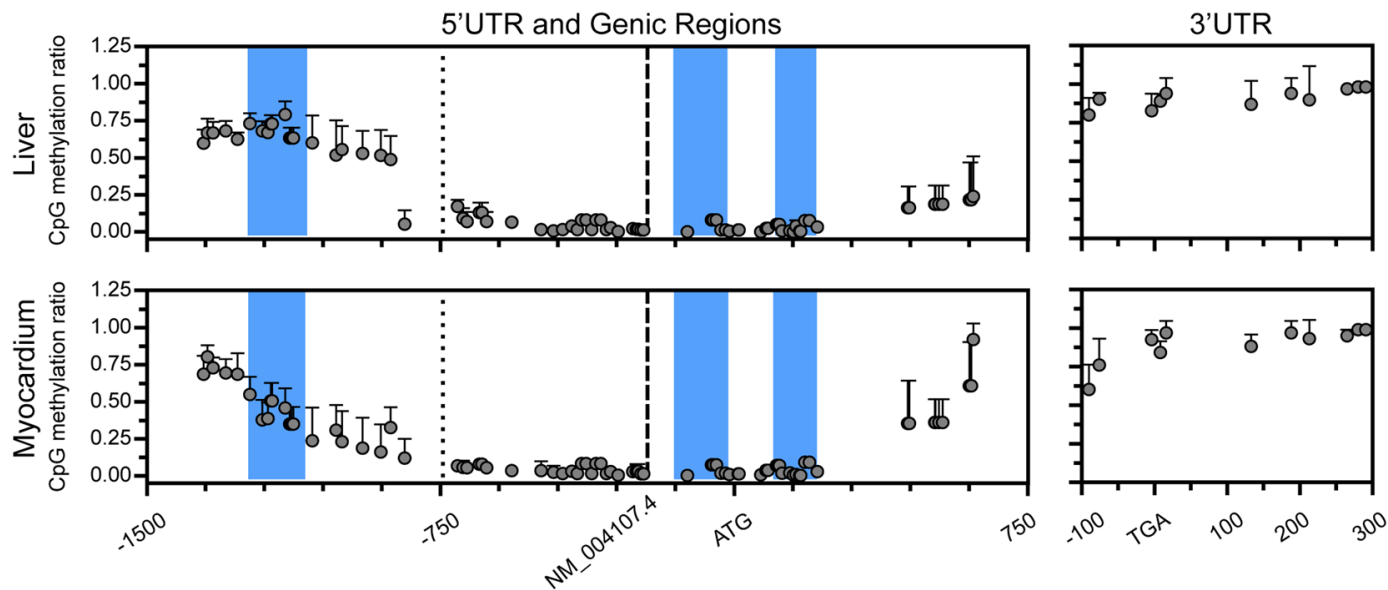

Figure 1. Quantitative DNA methylation analysis of the FCGRT locus in human tissues. Each point represents DNA methylation ratios \pm SD $(n=2-10)$ at individual $C p G$ sites in liver (upper panel) and myocardium (lower panel). Left panels represent the 5'UTR and partial genic regions of FCGRT. Right panels represent the $3^{\prime}$ UTR of FCGRT. Nucleotide positions are relative to the $\mathrm{A}_{+1} \mathrm{TG}$ (left panel) and $\mathrm{T}_{+1} \mathrm{GA}$ codons (right panel), respectively. Blue-shaded regions represent $\mathrm{CpG}$ islands. The dotted line represents the $-744 \mathrm{bp}$ reference site for "distal" and "proximal" $5^{\prime}$ UTR regions, and the dashed line represents the transcription start site for FCGRT transcript NM_004107.4(-222bp).

\section{Results}

FCGRT methylation in human liver and myocardium. Bioinformatics analysis of a $\approx 2.0 \mathrm{~Kb}$ region encompassing up to $1.4 \mathrm{~Kb}$ upstream and $0.6 \mathrm{~Kb}$ downstream the start $\mathrm{A}_{+1}$ TG codon of FCGRT revealed three distinct $\mathrm{CpG}$ islands $\left(\mathrm{CGI}_{\# 1}:-1241\right.$ to -1086 bp relative to $\mathrm{A}_{+1} \mathrm{TG}, \mathrm{CGI}_{\# 2}:-155$ to $-9 \mathrm{bp}$, and $\mathrm{CGI}_{\# 3}:+101$ to +218 bp) (Fig. 1). Quantitative DNA methylation analysis was performed to examine the extent of methylation in CpG sites located within the "distal" and "proximal" 5'UTR of FCGRT, as well as exon 1 and intron 1, in DNA samples from human liver and myocardium (Supplementary Table S1). The analysis encompassed approximately $82 \%$ (53 sites) of the possible CpG sites located within $1.4 \mathrm{~Kb}$ upstream the $\mathrm{A}_{+1} \mathrm{TG}$ codon (Fig. 1). In samples from both tissues, DNA methylation was most pronounced at $\mathrm{CpG}$ sites located within the distal portion of the 5'UTR, while DNA methylation was largely absent within the proximal region (Fig. 1). On average, the extent of DNA methylation in the distal 5'UTR of FCGRT was $58.7 \pm 12.9 \%$ in liver and $41.2 \pm 9.1 \%$ in myocardium (Fig. 1). The extent of DNA methylation within the proximal $5^{\prime}$ UTR was $4.6 \pm 3.9 \%$ in liver and $4.5 \pm 2.8 \%$ in myocardium (Fig. 1). DNA methylation analysis also included a total of $26 \mathrm{CpG}$ sites within a $610 \mathrm{bp}$ stretch downstream the $\mathrm{A}_{+1} \mathrm{TG}$ codon (exon 1 and intron 1) (Fig. 1). Within these genic regions the overall extent of CpG methylation was low and similar between both tissues (liver: $8.6 \pm 8.3 \%$, myocardium: $8.4 \pm 10.5 \%$ ) (Fig. 1). A total of $11 \mathrm{CpG}$ sites located near or within the $3^{\prime} \mathrm{UTR}\left(-90\right.$ to $+291 \mathrm{bp}$ relative to the stop codon, $\left.\mathrm{T}_{+1} \mathrm{GA}\right)$ were analyzed and showed high levels of methylation (liver: $93.4 \pm 4.4 \%$, myocardium: $94.1 \pm 5.3 \%$ ) (Fig. 1).

FCGRT mRNA expression in liver and myocardium. The expression of FCGRT mRNA (transcript NM_004107.4) in samples from liver and myocardium varied by approximately 7- and 14- fold, respectively (Fig. 2a). Correlation analyses between methylation levels and FCGRT mRNA expression were performed across all CpG sites located within the 5'UTR. In liver tissue, there was a significant negative correlation between methylation levels at CpG site $-707 \mathrm{bp}$ and FCGRT mRNA expression (Fig. 2b). In myocardium, there were negative correlations between the extent of methylation at CpG sites $-1017 \mathrm{bp},-903 \mathrm{bp}$, and $-842 \mathrm{bp}$ and FCGRT mRNA expression (Fig. 2b). The analysis was expanded by using DNA methylation and FGCRT mRNA expression data from 16 liver-derived cell lines available at the Cancer Cell Line Encyclopedia (Supplementary Table S1) (CCLE, https://portals.broadinstitute.org/ccle $)^{23}$. In liver-derived cell lines, there was a negative correlation between the extent of DNA methylation in the -1021 to $-628 \mathrm{bp}$ region of FCGRT and FCGRT mRNA expression $\left(\mathrm{R}^{2}=0.4201, P=0.0066\right)$ (Fig. $2 \mathrm{c}$ ).

Basal FcRn expression and FCGRT methylation in model human cell lines. Basal expressions of FCGRT mRNA and FcRn protein were evaluated in the human hepatic cell lines HepG2 and SNU-475 (hepatocellular carcinoma), and in the cardiomyocyte cell line AC16 (SV40 transformed) (Fig. 3). Under basal conditions, AC16 and SNU-475 cells expressed similar FCGRT mRNA levels (1.00 \pm 0.26 and $1.00 \pm 0.29$ relative fold, respectively), while HepG2 cells expressed the highest relative levels of FCGRT mRNA (34.59 \pm 12.99 relative fold) (Fig. 3a). The trends in FCGRT mRNA expression were paralleled by basal FcRn protein expression. That is, HepG2 cells exhibited the highest levels of FcRn expression (245 \pm 34 Arbitrary Units of fluorescence, AU) compared to AC16 (118 $\pm 5 \mathrm{AU})$ and SNU-475 (50 $\pm 11 \mathrm{AU})$ cells (Fig. 3b,c). Next, the extent of basal methylation in the FCGRT locus ( -1058 to -587 bp region) was analyzed by bisulfite DNA sequencing. In general, there was a negative correspondence between the total number of methylated CpG sites in each cell line (i.e., SNU-475: 14 methylated/15 total CpG sites, AC16: 9 methylated/15 total CpG sites, and HepG2: 1 methylated/15 CpG sites) and basal FCGRT mRNA and FcRn expression (Fig. 3d). 

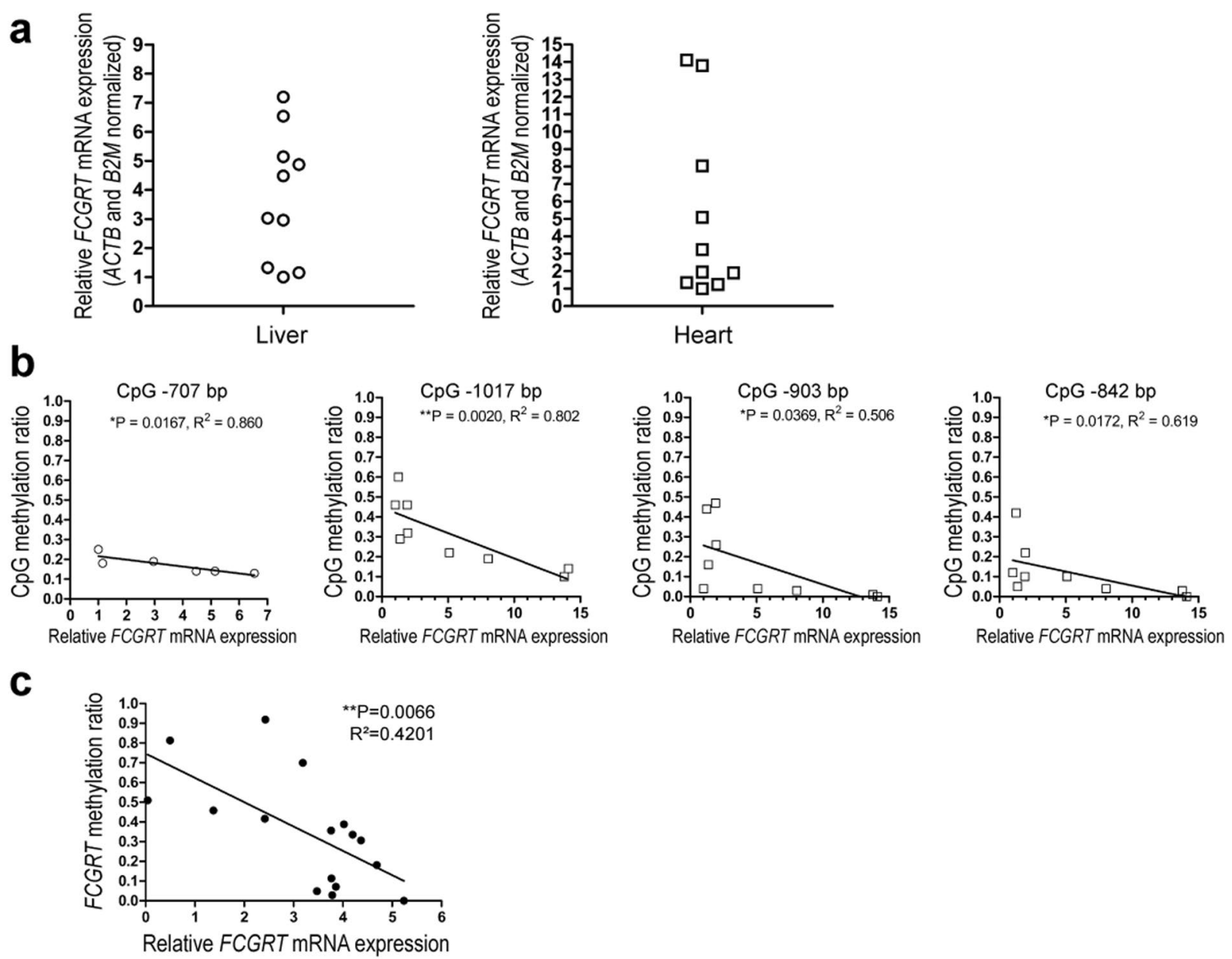

Figure 2. DNA methylation status and FCGRT expression correlation analysis in human liver and myocardium. (a) Relative FCGRT mRNA fold expression in samples from human liver $(\mathrm{n}=10)$ and myocardium $(\mathrm{n}=10)$. Each point represents the mean from two separate measurements performed in triplicates. (b) Linear regression analysis of methylation at individual CpG sites in DNA samples from liver (o) and myocardium (ם) versus FCGRT mRNA relative fold expression. Detection of the $-707 \mathrm{bp} \mathrm{CpG} \mathrm{site} \mathrm{failed} \mathrm{in} 4$ liver samples (o), and detection of CpG sites $-1017,-903$ and -842 bp was successful in 9 out of 10 myocardial samples (ם). (c) Linear regression analysis of DNA methylation ratios versus FCGRT mRNA relative expression in liver-derived cell lines $(\mathrm{n}=16$. Data from the Cancer Cell Line Encyclopedia database). Data show mean methylation ratios in the -1021 to -628 bp region of FCGRT. $* \mathrm{P}<0.05, * * \mathrm{P}<0.01$, Spearman rank-order correlation.

Impact of FCGRT methylation status on the expression of FCRn in model human cell lines. The impact of FCGRT gene methylation on the dynamics of FcRn expression was examined by treating HepG2, SNU475, and AC16 cells with the demethylating drug 5-Aza-2'-deoxycytidine (Aza). Treatments with $5 \mu \mathrm{M}$ Aza for 72 hours exerted negligible cytotoxicity in all cell lines (Supplementary Fig. S1). Aza treatment increased FCGRT mRNA expression in all cell lines (i.e., SNU-475: 80\%, AC16: 300\%, and HepG2: 40\%) (Fig. 4a). Fluorescence microscopy analysis showed that Aza treatments increased FcRn protein expression in SNU-475 cells (166\%) and AC16 cells (80\%), but not in HepG2 cells (Fig. 4b,c). Aza treatment modified CpG methylation status in the -1058 to -587 bp region of FCGRT (Fig. 5). In basal conditions, HepG2 cells showed the lowest levels of overall methylation in the -1058 to -587 bp region, AC16 cells displayed an intermediate degree of methylation, while SNU-475 exhibited the higher extent of constitutive CpG methylation (Table 1). Analysis of the extent of DNA demethylation after Aza treatment showed that in general, the FCGRT region including CpG sites from - 1021 to -842 bp was more sensitive to Aza treatment in SNU-475 and AC16 cells (Table 1). Specific CpG sites (i.e., AC16: $-1021 \mathrm{bp}$ and $-878 \mathrm{bp}$, and SNU-475: $-842 \mathrm{bp}$ ) were relatively more sensitive to the de-methylating effect of Aza (Fig. 5). Aza treatment for $120 \mathrm{~h}$ resulted in more pronounced changes in CpG methylation status and decreased cellular viability (Table 1, Supplementary Fig. S1).

Binding of transcription factors to the $5^{\prime}$ UTR of FCGRT. Bioinformatics analysis identified multiple potential binding sites for transcription factors to the $5^{\prime} \mathrm{UTR}$ of FCGRT (Fig. 6a). The region with differentially methylated $\mathrm{CpG}$ sites (i.e., -1058 to $-587 \mathrm{bp}$ ) contained potential binding sites for Sp1 transcription factor (Sp1), GA-binding protein alpha chain $(\mathrm{GABP} \alpha)$, and zinc finger and BTB domain-containing protein 7A 
a

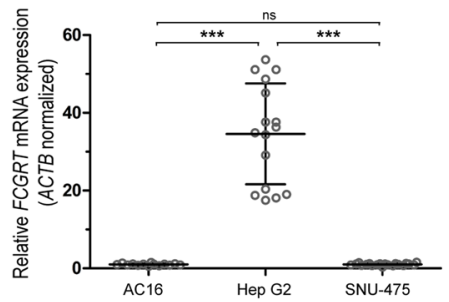

b

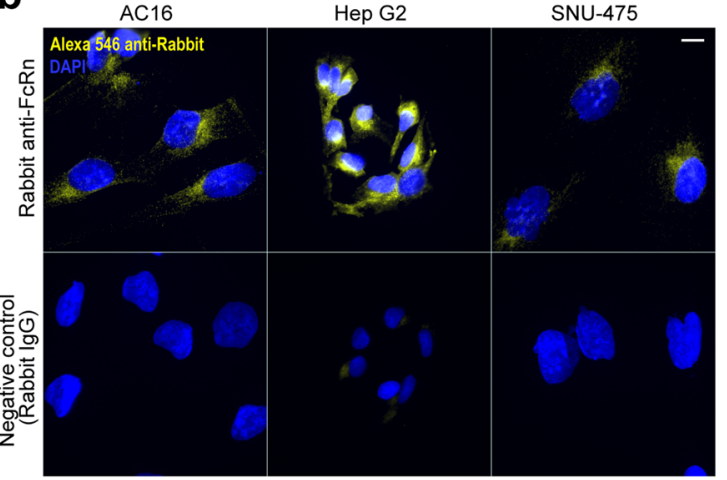

C

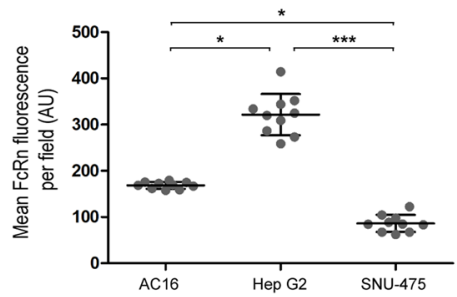

d

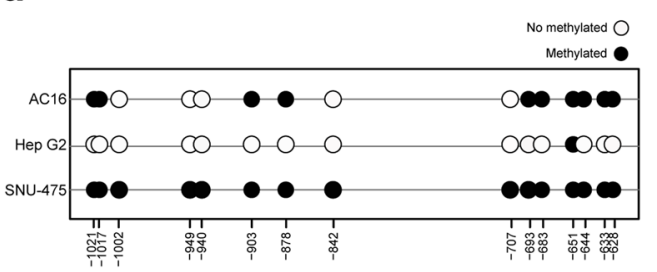

Figure 3. FCGRT expression and DNA methylation status in AC16, Hep G2, and SNU-475 cells. (a) Basal FCGRT mRNA relative fold expression. Each point represents individual measurements. Horizontal bars show the mean $\pm \mathrm{SD}$ from three determinations performed in quintuplicates. *** $\mathrm{P}<0.001$, ns $=$ not significant, 1-way ANOVA (Turkey's test). (b) Fluorescence microcopy analysis of basal FcRn expression (yellow). Nuclei were stained with DAPI (blue). Scale bar: $10 \mu \mathrm{m}$. (c) Quantitation of FcRn relative expression. Each point represents an individual measurement. Horizontal bars show the mean $\pm \mathrm{SD}$ (arbitrary units, $\mathrm{AU}$ ) from a representative experiment. *** $\mathrm{P}<0.001, * * \mathrm{P}<0.01$ ANOVA (Kruskal-Wallis test). (d) Bisulfite analysis of $\mathrm{CpG}$ sites methylation status in the -1058 to $-587 \mathrm{bp}$ region of FCGRT. Black circles represent methylated CpG sites ( $\geq 1$ positive clone), and clear circles represent demethylated CpG sites (no positive clones).

(Zbtb7a) (Fig. 6a). These transcription factors were selected for further analysis based on the following criteria: 1) ubiquitous expression according to the human protein atlas database (http://www.proteinatlas.org), 2) potential binding to the FCGRT target region according to MeDReaders (http://medreader.org), and 3) previous evidence of interactions with the FCGRT promoter region ${ }^{24-27}$. AC16 cardiomyocytes were selected to analyze the role of FCGRT methylation on the binding of candidate transcription factors because the cells: 1) display intermediate level of FCGRT methylation, and 2) are sensitive to Aza treatment. In AC16 cells, Sp1 and Zbtb7a bind to the $5^{\prime}$ UTR of FCGRT as suggested by chromatin immunoprecipitation experiments (ChIP), while GABP $\alpha$ showed no apparent binding to the same region (Fig. 6b,c). ChIP assays with primers spanning differentially methylated segments evidenced interactions between Sp1 and the -923 to -825 bp region $(30 \pm 17$-fold enrichment, primer set 2), and Zbtb7a and the -1045 to -825 bp region of FCGRT ( $23 \pm 3$-fold enrichment, primer set 1 , and $34 \pm 22$-fold enrichment, primer set 2) (Fig. 6c). No relevant interactions between any of the three transcription factors and the -729 to $-592 \mathrm{bp}$ region (primer set 3) were detected (Fig. 6b). No interactions between GABP $\alpha$, Sp1, or Zbtb7a, and the -1045 to -825 bp region were evident after treating AC16 cells with Aza ( $5 \mu \mathrm{M}$ for $72 \mathrm{~h}$ ) (Fig. 6b,c). Confirmatory dot-blot assays showed that $\mathrm{GABP} \alpha, \mathrm{Sp} 1$, and Zbtb7a were expressed in AC16 cells under basal conditions and after incubations with Aza (Supplementary Fig. S2).

\section{Discussion}

In this study, we investigated the role of DNA methylation as potential epigenetic regulator of human FCGRT expression. Quantitative DNA methylation analysis of the FCGRT locus revealed differentially methylated regions in DNA samples from liver and myocardium (Fig. 1). Seminal work by Mikulska and Simister showed that the -1529 to $-570 \mathrm{bp}$ region of FCGRT is essential for gene promoter activity ${ }^{26}$. This region exhibits variable levels of CpG methylation, and the extent of methylation in certain CpG sites (i.e., CpG sites: $-1017,-903,-842$, and $-707 \mathrm{bp}$ ) correlates with FCGRT mRNA expression in liver and myocardial tissues (Fig. 2a,b). In line, global methylation levels in the -1529 to -570 bp region correlates with FCGRT mRNA expression in cell lines derived from hepatocellular carcinomas (Fig. 2c).

In general, increased DNA methylation in gene regulatory regions decreases gene expression ${ }^{28,29}$. HepG2 cells exhibited low levels of FCGRT locus methylation and relatively higher levels of basal FCGRT mRNA and FcRn protein expression in comparison to AC16 and SNU-475 cells (Fig. 3). Treatments with the de-methylating drug Aza decreased the levels of FCGRT locus methylation in AC16 and SNU-475 cells which was paralleled by increases in FCGRT mRNA and FcRn expression (Figs 4 and 5). Thus, data from these model cell lines further suggest that differential methylation in the -1058 to -587 bp regulatory region of FCGRT contributes to $\mathrm{FcRn}$ expression in liver and myocardium. Liver and myocardium are tissues composed of different cell types that exhibit variable levels of FcRn expression. For example, Kupffer cells from human liver express relatively 
a
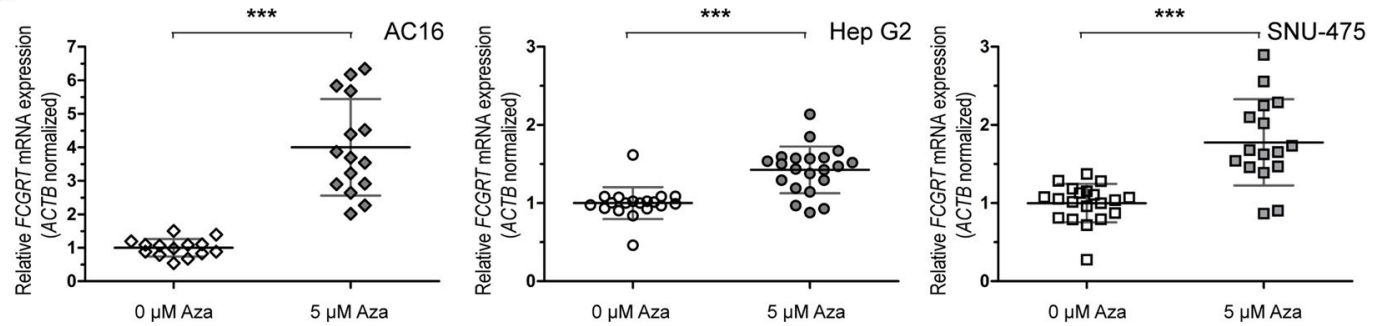

b

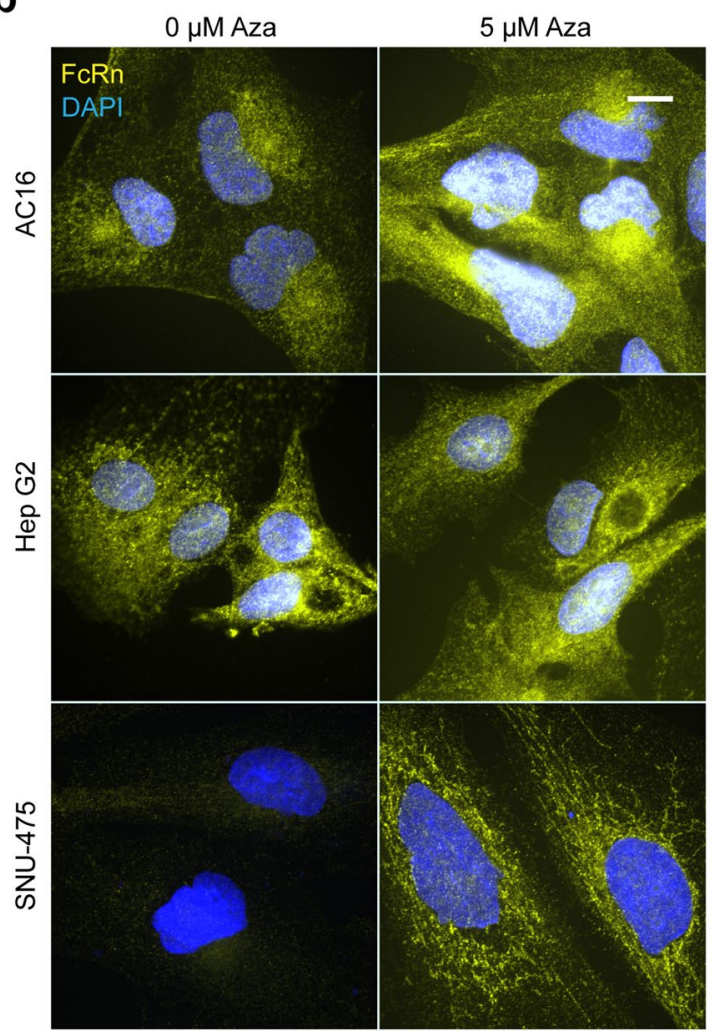

C
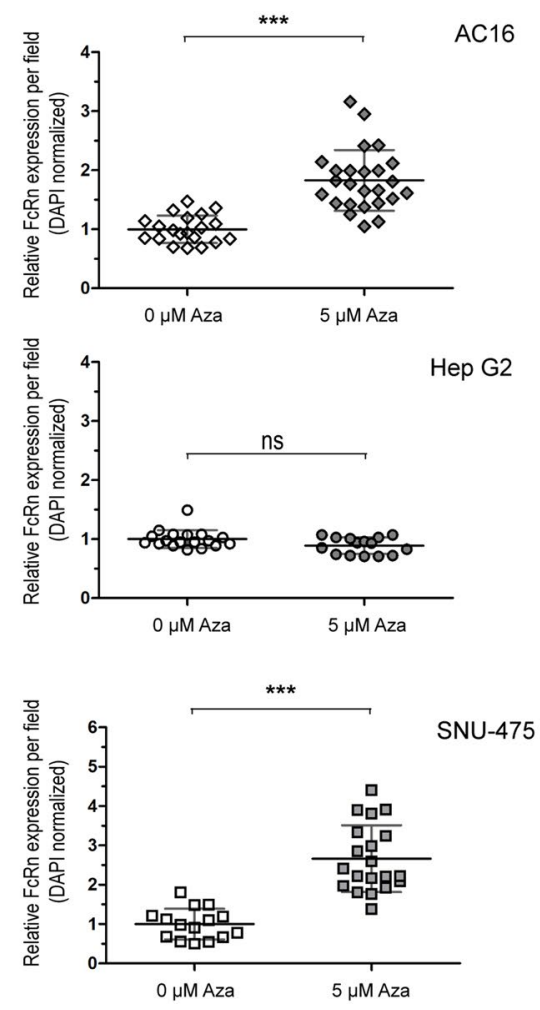

Figure 4. Impact of Aza treatment on FCGRT expression. (a) FCGRT mRNA expression in AC16, Hep G2, and SNU-475 cells after Aza treatment $(5 \mu \mathrm{M}, 72 \mathrm{~h})$. Plots depict fold differences in FCGRT mRNA expression levels relative to controls $(0 \mu \mathrm{M}$ Aza $)$. Each point represents individual measurements. Horizontal bars show the mean \pm SD from three experiments performed in quintuplicates. $* * * \mathrm{P}<0.001$, ns $=$ not significant, Student's t test. (b) Cellular FcRn protein expression (yellow) before and after Aza treatment ( $5 \mu \mathrm{M}, 72 \mathrm{~h}$. Representative images). Nuclei were stained with DAPI (blue). Scale bar: $10 \mu \mathrm{m}$. (c) Quantitation of FcRn relative fold expression after Aza treatments. Each point represents individual measurements. Horizontal bars show the mean \pm SD from two independent experiments ( $8-10$ fields per condition). $* * * \mathrm{P}<0.001$, ns $=$ not significant, Student's t test.

high levels of FcRn in comparison to sinusoidal endothelial cells ${ }^{30}$. Thus, future studies should aim to elucidate the contribution of differential FCGRT methylation to the dynamics of FcRn expression in individual cell types from relevant tissues. Furthermore, epigenetically active substances such as 5-azacytidine and 5-aza-2' deoxycytidine are used in the clinic for the therapy of myelodysplastic syndromes (MDS) and acute myeloid leukemia $(\mathrm{AML})^{31,32}$. Since Aza increases the expression of FcRn in model cell lines, it will be of interest to evaluate whether Aza treatment modifies a) the pharmacological behavior (e.g., pharmacokinetics) of Fc-containing monoclonal antibody drugs, and b) albumin tumor consumption in the context of therapy for AML/MDS ${ }^{33,34}$.

Relatively few regulatory elements within the putative promoter region of human FCGRT have been characterized to date. Sachs et al. demonstrated that a polymorphic VNTR in the FCGRT promoter influences FcRn expression $^{17}$. Mikulska showed that Sp1, Sp2, Sp3, c-Fos, c-Jun, YY1, C/EBP $\beta$ and C/EBP $\Delta$ interact with distinct regions in the FCGRT promoter ${ }^{26,35}$. Our results in AC16 cells suggest that Zbtb7a and Sp1 bind to overlapping methylated regions in the FCGRT promoter (Fig. 6). Zbtb7a and Sp1 interactions with FCGRT were abolished with Aza treatment (Fig. 6). Zbtb7a recognizes methylated CpGs and represses transcription by mechanisms that include prevention of transcription factor binding to DNA and recruitment of corepressors and histone deacetylase (HDAC) complexes ${ }^{36-38}$. Sp1 can recognize methylated CpG sites, and Zbtb7a is capable of interacting 


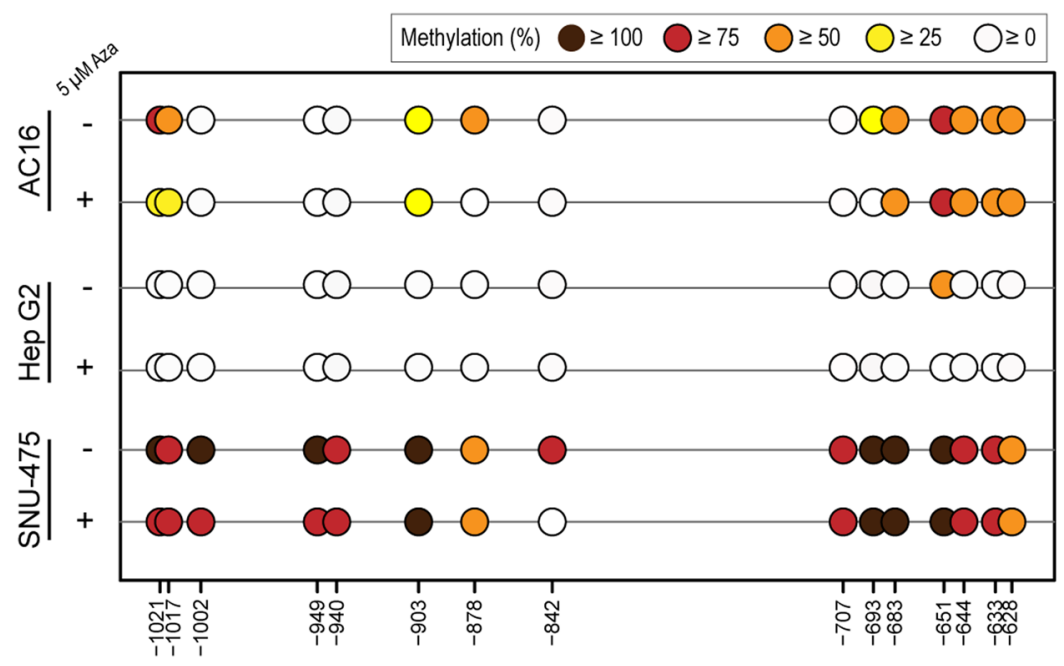

Figure 5. Effect of Aza treatment on FCGRT DNA methylation. Methylation levels at individual CpG sites in the -1058 to $-587 \mathrm{bp}$ region of FCGRT after Aza treatment $(5 \mu \mathrm{M}, 72 \mathrm{~h})$. Circles represent individual CpG sites. Mean methylation level (\%) at individual CpG sites were determined by bisulfite sequencing.

\begin{tabular}{|c|c|c|c|c|c|c|c|c|c|}
\hline \multirow[b]{2}{*}{ Cell line } & \multicolumn{3}{|c|}{ CpG sites in -1021 to -842 bp region } & \multicolumn{3}{|c|}{ CpG sites in -707 to -628 bp region } & \multicolumn{3}{|c|}{ CpG sites in -1021 to -628 bp region } \\
\hline & Basal & $\begin{array}{l}5 \mu \mathrm{M} \mathrm{Aza} \\
72 \mathrm{~h}\end{array}$ & $\begin{array}{l}5 \mu \mathrm{M} \text { Aza } \\
120 \mathrm{~h}\end{array}$ & Basal & $\begin{array}{l}5 \mu \mathrm{M} \text { Aza } \\
72 \mathrm{~h}\end{array}$ & $\begin{array}{l}5 \mu \mathrm{M} \text { Aza } \\
120 \mathrm{~h}\end{array}$ & Basal & $\begin{array}{l}5 \mu \mathrm{M} \text { Aza } \\
72 \mathrm{~h}\end{array}$ & $\begin{array}{l}5 \mu \mathrm{M} \text { Aza } \\
120 \mathrm{~h}\end{array}$ \\
\hline $\mathrm{AC} 16$ & $25 \pm 28 \%$ & $9 \pm 12 \%$ & $0 \pm 0 \%$ & $46 \pm 22 \%$ & $41 \pm 27 \%$ & $21 \pm 9 \%$ & $35 \pm 27 \%$ & $24 \pm 26 \%$ & $10 \pm 12 \%$ \\
\hline Hep G2 & $0 \pm 0 \%$ & $0 \pm 0 \%$ & $0 \pm 0 \%$ & $7 \pm 17 \%$ & $0 \pm 0 \%$ & $0 \pm 0 \%$ & $3 \pm 12 \%$ & $0 \pm 0 \%$ & $0 \pm 0 \%$ \\
\hline SNU-475 & $84 \pm 17 \%$ & $69 \pm 27 \%$ & $0 \pm 0 \%$ & $80 \pm 19 \%$ & $82 \pm 19 \%$ & $32 \pm 17 \%$ & $83 \pm 18 \%$ & $75 \pm 25 \%$ & $15 \pm 20 \%$ \\
\hline
\end{tabular}

Table 1. DNA methylation (\%) in the FCGRT locus before and after Aza treatment.

with Sp1 which results in transcriptional repression due to interference of Sp1's DNA binding activity ${ }^{37,39,40}$. Thus, ChIP data may reflect the presence of a Sp1-Zbtb7a complex bound to the -923 to -825 bp region of FCGRT. This region is relatively more sensitive to demethylation by Aza in cells. Interindividual levels of methylation in liver and myocardium are also more variable in this region and this variability may be relevant for the epigenetic control of FCGRT expression. Additional studies are necessary in order to elucidate the role of FCGRT methylation on the binding of Zbtb7a, Sp1, and other transcription factors in various cell types and tissues at different diseases states.

Our results suggest that differential DNA methylation in specific regions of the FCGRT gene promoter contributes to regulate the expression of FcRn. This study provides a foundation to further define the contribution of epigenetic factors during the control of FcRn expression and IgG traffic in human tissues.

\section{Methods}

Human tissue samples. The Institutional Review Board of the State University of New York at Buffalo (UB-IRB) approved this research. UB-IRB determined that this research is not research with human subjects. This research meets exempt criteria, 45 CRF 46,101(b)(4). Human liver $(n=10)$ and myocardial $(n=10)$ tissue samples were provided by the National Disease Research Interchange (NDRI), the Cooperative Human Tissue Network (CHTN), and the Liver Tissue Procurement and Distribution System (LTPDS, National Institutes of Health Contract N01-DK-9-2310). As per Federal and State regulations, CHTN, NDRI, and LTPDS require every procurement site to obtain informed consent in compliance with all regulations governing that process in writing from any donor of human tissue (or the next of kin thereof) for the use of tissue for research. Each signed consent form is kept on file at the tissue acquisition site and information is never released to any third party. CHTN, NDRI, and LTPDS assign computer generated codes to each donor and do not maintain any information that could be used to identify the donor. CHTN, NDRI, and LTPDS provide anonymous samples coded with unique sample identification numbers. Procurement protocols for this project were reviewed and approved by NDRI, CHTN, and LTPDS.

Cell culture and 5-Aza-2'-deoxycytidine treatments. AC16 (Millipore Sigma), HepG2 (American Type Culture Collection, ATCC), and SNU-475 (ATCC) were cultured using DMEM/F12, DMEM, and RPMI, respectively (Life Technologies), supplemented with $10 \%(\mathrm{v} / \mathrm{v})$ fetal bovine serum (FBS) in standard incubation conditions at $37^{\circ} \mathrm{C}, 5 \% \mathrm{CO}_{2}$, and $95 \%$ relative humidity. 
a

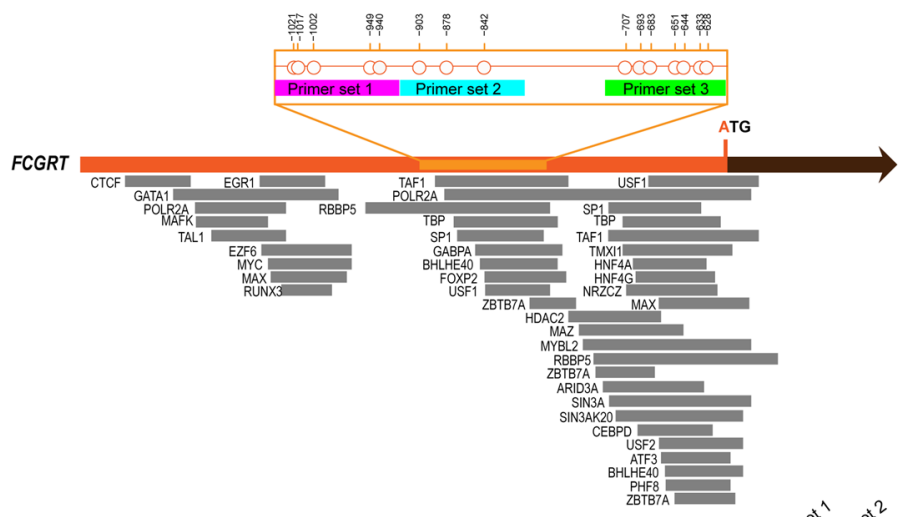

b

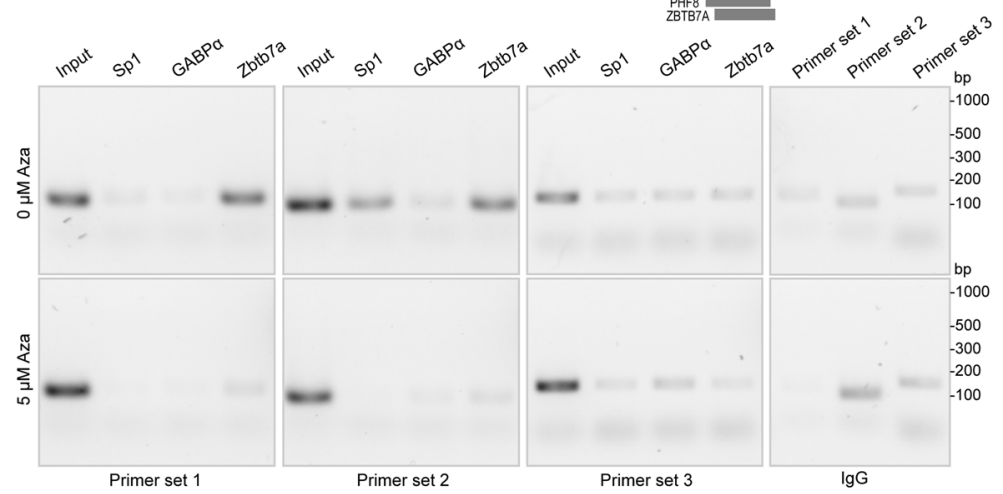

C
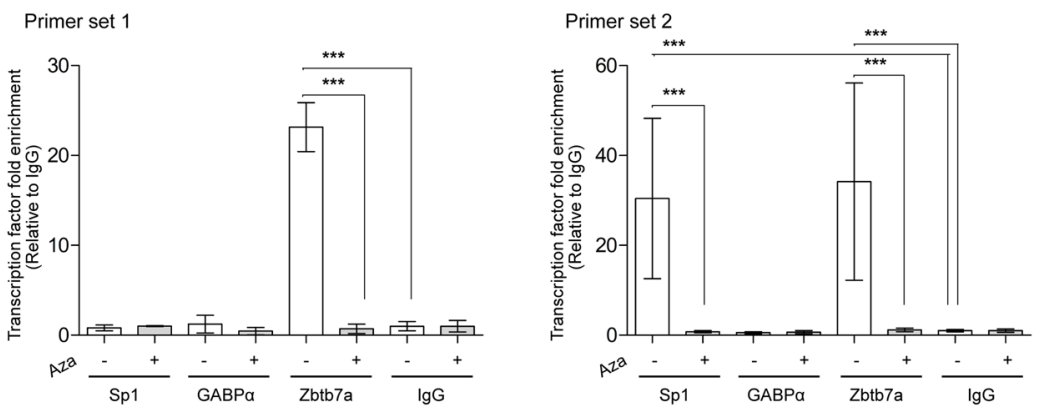

Figure 6. Role of DNA methylation on the binding of transcription factors to FCGRT. (a) Bioinformatics analysis of transcription factors potentially targeting the FCGRT locus ( -1793 bp to $\mathrm{A}_{+1}$ TG region). The $-1058 \mathrm{bp}$ to $\mathrm{A}_{+1} \mathrm{TG}$ region is shown in orange. Regions amplified by primer sets 1, 2, and 3 are indicated in magenta, cyan and green, respectively. $\mathrm{CpG}$ sites are represented as circles. (b) Chromatin immunoprecipitation (ChIP) assays evaluating the binding of Sp1, GABP $\alpha$, and Zbtb7a, to the FCGRT locus in AC16 cells with or without Aza treatment $(5 \mu \mathrm{M}, 72 \mathrm{~h})$. Panels show representative agarose gel electrophoresis analysis of PCR amplification products obtained with primers sets 1-3 using input samples and immunoprecipitated DNA samples. IgG controls are also shown (right panels, top, and bottom). Images were cropped from pictures taken from 3 individual agarose gels (full length pictures are included in supplementary material file 1, Figure S3). (c) Quantitative PCR analysis of ChIP samples assessing transcription factor enrichment in FCGRT regions amplified by primer sets 1 and 2. Values are expressed relative to values from IgG control antibody. Data show the mean $\pm \mathrm{SD}$ from two independent qPCR experiments (3-4 replicates). $* * * \mathrm{P}<0.0001,1$-way ANOVA (Turkey's test).

Cells were seeded $24 \mathrm{~h}$ before 5-Aza-2'-deoxycytidine (Aza, Sigma-Aldrich) treatments. Aza was added to fresh culture media every $24 \mathrm{~h}$ for a total of $72 \mathrm{~h}$. Cell viability was determined with the CellTiter-Glo Luminescent Viability Kit (Promega), according to the manufacturer's instructions.

DNA methylation analysis. Genomic DNA from tissues was extracted using the E.Z.N.A. Tissue DNA kit (Omega Bio-tek). Quantitative DNA methylation analysis of the FCGRT locus was performed with the EpiTYPER Mass Array System (Agena Bioscience) at the Genomics Shared Resource at Roswell Park Comprehensive Cancer Center (Buffalo, New York). Specific primer sets were used for amplification (Table S1). For each amplicon, standards consisting of $0 \%, 50 \%$, and $100 \%$ methylated DNA were used as calibrators. The relative location of individual $\mathrm{CpG}$ sites was determined by using the translation start site $\mathrm{A}_{+1} \mathrm{TG}$ of exon 1 as the origin. The relative location of CpG sites in the $3^{\prime} \mathrm{UTR}$ of FCGRT was determined by using the stop codon $\left(\mathrm{T}_{+1} \mathrm{GA}\right)$ as the origin. 
Quantitative real-time polymerase chain reaction. Total RNA was isolated from cells using Trizol reagent following the manufacturer's instructions (Thermo Fisher). FCGRT mRNA expression was analyzed with specific primers (Table S2). Total RNA (12.5 ng) was reverse transcribed and amplified with the iTaq Universal SYBR Green One-Step Kit (Bio-Rad). FCGRT and reference genes ( $A C T B$ and $B 2 M$ ) were amplified in parallel in a CFX96 Touch Real-Time PCR Detection System (Bio-Rad) with the following cycling parameters: $50^{\circ} \mathrm{C}$ for $10 \mathrm{~min}$ (reverse transcription), $95^{\circ} \mathrm{C}$ for $1 \mathrm{~min}$, followed by 44 cycles of $95^{\circ} \mathrm{C}$ for $10 \mathrm{~s}, 60.5^{\circ} \mathrm{C}$ for $20 \mathrm{~s}$. Calibration curves were prepared to analyze linearity and PCR efficiency. qRT-PCR data were analyzed using the $\Delta \Delta \mathrm{Ct}$ method with CFX manager Software (Bio-Rad). The $\Delta \mathrm{Ct}$ method was utilized for determining the relative abundance of FCGRT mRNA.

Fluorescence microscopy. Cells were grown on glass coverslips, fixed in $4 \%$ paraformaldehyde in phosphate-buffered saline (PBS) for $20 \mathrm{~min}$ at $4{ }^{\circ} \mathrm{C}$ and permeabilized with $0.1 \%$ Triton X-100 and $200 \mathrm{mM}$ glycine in PBS for $2 \mathrm{~min}$ at $4^{\circ} \mathrm{C}$, and washed with PBS. Samples were blocked in $3 \%$ bovine serum albumin (BSA)-PBS for $60 \mathrm{~min}$ at room temperature and incubated with rabbit anti-FcRn antibody (1/100, ab193148, Abcam) $2 \mathrm{~h}$ at room temperature followed by Alexa 546-conjugated anti-rabbit IgG (1/1,000; Invitrogen) for $1 \mathrm{~h}$. Negative controls were performed by replacing the anti-FcRn antibody with rabbit IgG (ab171870, Abcam) at the same concentration during immunostaining. Controls for secondary antibody immunostaining specificity without primary antibody were also included. Nuclei were stained with DAPI. Samples were mounted onto glass slides using FluorSave (Calbiochem). Images (12 bits) were obtained with a monochrome digital camera (Carl Zeiss, Axiocam MRm) attached to a fluorescence microscope (Carl Zeiss, Axiovert $200 \mathrm{M}$ ) using PlanApoN $60 \times 1.40$ NA oil immersion objective. Identical microscope configuration and camera settings were maintained during image acquisition for conditions from the same experiment. Images from multiple fields ( 10 fields/condition) were taken.

For FcRn quantification, images were processed using identical parameters with the Fiji software ${ }^{41}$. Images corresponding to different conditions were grouped in image stacks for further processing and comparisons. Background subtraction was identical for each stack, mean fluorescence in the 546 channel (corresponding to FcRn or negative controls) was measured by thresholding with the Huang method in Fiji, and signals from negative controls were then subtracted. Mean DAPI fluorescence signal was measured in the same way.

Bisulfite DNA sequencing. DNA from cells was extracted using the E.Z.N.A. Tissue DNA kit and converted with bisulfite using the MethylEdge Bisulfite Conversion System (Promega). Amplicons corresponding to the FCGRT 5'UTR were amplified using ZymoTaq PreMix (Zymo Research) and specific primers (Table S2). Amplicons were cloned into the pCR4-TOPO vector and transformed into TOP10 chemically competent $E$. Coli following the manufacturer's instructions (Thermo Fisher Sci.). Individual clones $(\mathrm{n}=4-8)$ for each amplicon were selected, and grown in LB broth supplemented with ampicillin (Sigma-Aldrich). Plasmid DNA was extracted from each clone using the PureYield Plasmid Miniprep System (Promega) and directly sequenced at the Genomics Shared Resource at the Roswell Park Comprehensive Cancer Center (Buffalo, New York).

Chromatin immunoprecipitation. ChIP assays were performed using the ChIP-IT High Sensitivity kit (53040, Active Motif) according to the manufacturer's instructions. Briefly, AC16 cells were cross-linked with 1\% formaldehyde at room temperature for $5 \mathrm{~min}$, repeatedly washed with ice-cold PBS, and lysed using a Dounce homogenizer followed by centrifugation. Chromatin was fragmented using enzymatic shearing. A fraction of the mixture of protein-DNA complex was used as "input DNA". Sheared chromatin $(1 \mu \mathrm{g})$ was then incubated overnight at $4{ }^{\circ} \mathrm{C}$ with $5 \mu \mathrm{g}$ of rabbit anti-GABP $\alpha$ antibody (PA5-27735, Invitrogene), rabbit anti-Sp1 antibody (sc-14027-X, Santa Cruz Biotechnology), rabbit anti-Zbtb7a antibody (A300-548A, Bethyl Laboratories) or normal rabbit IgG (ab171870, Abcam). Immuno-precipitated DNA was eluted using protein G agarose beads, then the cross-linking was reversed and the DNA was purified.

Recovered DNA samples and inputs were analyzed by PCR and qPCR. Three fragments (123, 99, and $138 \mathrm{bp})$ corresponding to the FCGRT promoter region were amplified with primers listed in Table S2. PCR products were resolved on a $\%$ agarose gel stained with SYBR Safe (Thermo Fisher Scientific), and visualized under UV light. For qPCR, DNA samples were amplified with the iTaq Universal SYBR Green One-Step Kit (Bio-Rad) in a CFX96 Touch Real-Time PCR Detection System (Bio-Rad) with the following cycling parameters: $95^{\circ} \mathrm{C}$ for $1 \mathrm{~min}$, followed by $35 \mathrm{cycles}$ of $95^{\circ} \mathrm{C}$ for $30 \mathrm{~s}, 58^{\circ} \mathrm{C}$ for $30 \mathrm{~s}$. The amount of DNA in each sample was extrapolated from standard curves for each PCR reaction, and fold enrichment values for target DNA sequences relative to IgG controls samples were determined.

Dot-blot assay. AC16 chromatin samples were spotted on nitrocellulose membranes (88018, Thermo Fisher Scientific) and dried at room temperature. Samples were blocked in $0.05 \%$ Tween $20-1 \%$ BSA-PBS for $30 \mathrm{~min}$ at room temperature in PBS, followed by incubation with anti- $\beta$-Actin (1/1000), anti-GABP $\alpha(1 / 250)$, anti-Sp1 (1/100) or anti-Zbtb7a (1/250) or without primary antibody in $0.05 \%$ Tween $20-0.1 \%$ BSA-PBS for $2 \mathrm{~h}$ at room temperature. Membranes were washed in $0.05 \%$ Tween 20 -PBS for 5 minutes and then probed with HRP-conjugated goat anti-rabbit secondary antibody (1/2000, Santa Cruz) in $0.05 \%$ Tween 20 -PBS for 30 min at room temperature. After washing, membranes were incubated during $1 \mathrm{~min}$ with Pierce ECL Substrate (Thermo Fisher Scientific) and imaged in a ChemiDoc XRS+ Imager (Bio Rad).

Densitometric analysis was performed with the Fiji software ${ }^{41}$. A region of interest (ROI) corresponding to the spotted region was manually selected, and the mean gray value was measured. Negative control signal was subtracted from all conditions and normalized to $\beta$-actin signal.

Bioinformatics. FCGRT reference sequence NM_004107.4 from the National Center for Biotechnology Information (Gene ID: 2217) was used for analysis. Predictions of CpG islands were performed with MethPrimer 
(http://www.urogene.org/cgi-bin/methprimer/methprimer.cgi) with the following standard criteria: island size $>100 \mathrm{bp}, \mathrm{CG} \%>50$ and $\mathrm{O} / \mathrm{E}$ ratio $>0.6^{42}$.

The analysis of transcription factors potentially targeting the -1793 bp to $\mathrm{A}_{+1}$ TG in the FCGRT locus was performed by incorporating ChIP-Seq datasets available from the Encode Project (https://www.encodeproject.org) and datasets available in Factorbook (http://www.factorbook.org/). These datasets were aggregated and viewed in the UCSC Genome Browser (https://genome.ucsc.edu/).

Data processing and statistical analysis. Data processing was performed with Excel 2016 (Microsoft Office). Statistical analyses were performed with GraphPad Prism version 7. The D'Agostino \& Pearson omnibus normality test was used to determine the normality of data sets. Comparisons between the means of two groups were performed with the Student's t-test or Mann-Whitney's U test for sets with normal and non-normal distributions, respectively. ANOVA with Tukey's or the Kruskal-Wallis test were used to analyze differences between means from multiple groups. Spearman's rank-order test was used for correlation analyses.

\section{Data Availability}

The datasets generated during the current study are available from the corresponding author upon request.

\section{References}

1. Praetor, A. \& Hunziker, W. beta(2)-Microglobulin is important for cell surface expression and pH-dependent IgG binding of human FcRn. J Cell Sci 115, 2389-2397 (2002).

2. Dickinson, B. L. et al. Bidirectional FcRn-dependent IgG transport in a polarized human intestinal epithelial cell line. J Clin Invest 104, 903-911, https://doi.org/10.1172/JCI6968 (1999).

3. Antohe, F., Radulescu, L., Gafencu, A., Ghetie, V. \& Simionescu, M. Expression of functionally active FcRn and the differentiated bidirectional transport of IgG in human placental endothelial cells. Hum Immunol 62, 93-105, doi:S0198885900002445 (2001).

4. Ober, R. J., Martinez, C., Vaccaro, C., Zhou, J. \& Ward, E. S. Visualizing the site and dynamics of IgG salvage by the MHC class I-related receptor, FcRn. J Immunol 172, 2021-2029 (2004).

5. Anderson, C. L. et al. Perspective-FcRn transports albumin: relevance to immunology and medicine. Trends Immunol 27, 343-348, https://doi.org/10.1016/j.it.2006.05.004 (2006).

6. Borrok, M. J. et al. pH-dependent binding engineering reveals an FcRn affinity threshold that governs IgG recycling. J Biol Chem 290, 4282-4290, https://doi.org/10.1074/jbc.M114.603712 (2015).

7. Pyzik, M. et al. Hepatic FcRn regulates albumin homeostasis and susceptibility to liver injury. Proc Natl Acad Sci USA 114, E2862-E2871, https://doi.org/10.1073/pnas.16182911141618291114 (2017).

8. Bonilla, F. A. Pharmacokinetics of immunoglobulin administered via intravenous or subcutaneous routes. Immunol Allergy Clin North Am 28, 803-819, ix, https://doi.org/10.1016/j.iac.2008.06.006 (2008).

9. Mendez, C. M., McClain, C. J. \& Marsano, L. S. Albumin therapy in clinical practice. Nutr Clin Pract 20, 314-320 (2005).

10. Robbie, G. J. et al. A novel investigational Fc-modified humanized monoclonal antibody, motavizumab-YTE, has an extended halflife in healthy adults. Antimicrob Agents Chemother 57, 6147-6153, https://doi.org/10.1128/AAC.01285-13, (2013).

11. Liu, L. Pharmacokinetics of monoclonal antibodies and Fc-fusion proteins. Protein \& cell. https://doi.org/10.1007/s13238-017-04084 (2017).

12. Dalloneau, E. et al. Downregulation of the neonatal Fc receptor expression in non-small cell lung cancer tissue is associated with a poor prognosis. Oncotarget 7, 54415-54429, https://doi.org/10.18632/oncotarget.10074 (2016).

13. de Souza, E. G. et al. Maternal-Foetal Diabetes Modifies Neonatal Fc Receptor Expression on Human Leucocytes. Scandinavian journal of immunology 84, 237-244, https://doi.org/10.1111/sji.12466 (2016).

14. Cianga, P., Cianga, C., Cozma, L., Ward, E. S. \& Carasevici, E. The MHC class I related Fc receptor, FcRn, is expressed in the epithelial cells of the human mammary gland. Human immunology 64, 1152-1159 (2003).

15. Liu, X. et al. Activation of the JAK/STAT-1 signaling pathway by IFN-gamma can down-regulate functional expression of the MHC class I-related neonatal Fc receptor for IgG. J Immunol 181, 449-463 (2008).

16. Liu, X. et al. NF-kappaB signaling regulates functional expression of the MHC class I-related neonatal Fc receptor for IgG via intronic binding sequences. J Immunol 179, 2999-3011 (2007).

17. Sachs, U. J. et al. A variable number of tandem repeats polymorphism influences the transcriptional activity of the neonatal Fc receptor alpha-chain promoter. Immunology 119, 83-89, https://doi.org/10.1111/j.1365-2567.2006.02408.x (2006).

18. Ferguson, D. C. \& Blanco, J. G. Regulation of the Human Fc-Neonatal Receptor alpha-Chain Gene FCGRT by MicroRNA-3181. Pharm Res 35, 15, https://doi.org/10.1007/s11095-017-2294-0, (2018).

19. Hashimshony, T., Zhang, J., Keshet, I., Bustin, M. \& Cedar, H. The role of DNA methylation in setting up chromatin structure during development. Nat Genet 34, 187-192, https://doi.org/10.1038/ng1158ng1158 (2003).

20. Jones, P. A. Functions of DNA methylation: islands, start sites, gene bodies and beyond. Nat Rev Genet 13, 484-492, https://doi. org/10.1038/nrg3230nrg3230 (2012).

21. Tirado-Magallanes, R., Rebbani, K., Lim, R., Pradhan, S. \& Benoukraf, T. Whole genome DNA methylation: beyond genes silencing. Oncotarget 8, 5629-5637, https://doi.org/10.18632/oncotarget.1356213562 (2017).

22. Blattler, A. \& Farnham, P. J. Cross-talk between site-specific transcription factors and DNA methylation states. J Biol Chem 288, 34287-34294, https://doi.org/10.1074/jbc.R113.512517R113.512517 (2013).

23. Barretina, J. et al. The Cancer Cell Line Encyclopedia enables predictive modelling of anticancer drug sensitivity. Nature 483, 603-607, https://doi.org/10.1038/nature11003 (2012).

24. Wang, G. et al. MeDReaders: a database for transcription factors that bind to methylated DNA. Nucleic Acids Res 46, D146-D151, https://doi.org/10.1093/nar/gkx1096 (2018).

25. Uhlen, M. et al. Proteomics. Tissue-based map of the human proteome. Science 347, 1260419, https://doi.org/10.1126/ science.1260419 (2015)

26. Mikulska, J. E. \& Simister, N. E. Analysis of the promoter region of the human FcRn gene. Biochim Biophys Acta 1492, 180-184 (2000).

27. Tiwari, B. \& Junghans, R. P. Functional analysis of the mouse Fcgrt 5' proximal promoter. Biochim Biophys Acta 1681, 88-98, https:// doi.org/10.1016/j.bbaexp.2004.10.002 (2005).

28. Mohn, F. et al. Lineage-specific polycomb targets and de novo DNA methylation define restriction and potential of neuronal progenitors. Mol Cell 30, 755-766, https://doi.org/10.1016/j.molcel.2008.05.007 (2008).

29. Moore, L. D., Le, T. \& Fan, G. DNA methylation and its basic function. Neuropsychopharmacology 38, 23-38, https://doi.org/10.1038/ npp.2012.112npp2012112 (2013).

30. Latvala, S., Jacobsen, B., Otteneder, M. B., Herrmann, A. \& Kronenberg, S. Distribution of FcRn Across Species and Tissues. J Histochem Cytochem 65, 321-333, https://doi.org/10.1369/0022155417705095 (2017). 
31. Issa, J. P. et al. Phase 1 study of low-dose prolonged exposure schedules of the hypomethylating agent 5-aza-2'-deoxycytidine (decitabine) in hematopoietic malignancies. Blood 103, 1635-1640, https://doi.org/10.1182/blood-2003-03-0687 (2004).

32. Silverman, L. R. et al. Randomized controlled trial of azacitidine in patients with the myelodysplastic syndrome: a study of the cancer and leukemia group B. J Clin Oncol 20, 2429-2440, https://doi.org/10.1200/JCO.2002.04.117 (2002)

33. Kadia, T. M., Ravandi, F., Cortes, J. \& Kantarjian, H. New drugs in acute myeloid leukemia. Ann Oncol 27, 770-778, https://doi. org/10.1093/annonc/mdw015 (2016).

34. Swiercz, R. et al. Loss of expression of the recycling receptor, FcRn, promotes tumor cell growth by increasing albumin consumption. Oncotarget 8, 3528-3541, https://doi.org/10.18632/oncotarget.13869 (2017).

35. Mikulska, J. E. Analysis of Response Elements Involved in the Regulation of the Human Neonatal Fc Receptor Gene (FCGRT). PLoS One 10, e0135141, https://doi.org/10.1371/journal.pone.0135141 (2015).

36. Choi, W. I. et al. The proto-oncoprotein FBI-1 interacts with MBD3 to recruit the Mi-2/NuRD-HDAC complex and BCoR and to silence p21WAF/CDKN1A by DNA methylation. Nucleic Acids Res 41, 6403-6420, https://doi.org/10.1093/nar/gkt359 (2013).

37. Yin, Y. et al. Impact of cytosine methylation on DNA binding specificities of human transcription factors. Science 356, https://doi. org/10.1126/science.aaj2239 (2017).

38. Maeda, T. et al. Role of the proto-oncogene Pokemon in cellular transformation and ARF repression. Nature 433, 278-285, https:// doi.org/10.1038/nature03203 (2005)

39. Lee, D. K., Suh, D., Edenberg, H. J. \& Hur, M. W. POZ domain transcription factor, FBI-1, represses transcription of ADH5/FDH by interacting with the zinc finger and interfering with DNA binding activity of Sp1. J Biol Chem 277, 26761-26768, https://doi. org/10.1074/jbc.M202078200 (2002).

40. Holler, M., Westin, G., Jiricny, J. \& Schaffner, W. Sp1 transcription factor binds DNA and activates transcription even when the binding site is CpG methylated. Genes Dev 2, 1127-1135 (1988).

41. Schindelin, J. et al. Fiji: an open-source platform for biological-image analysis. Nat Methods 9, 676-682, https://doi.org/10.1038/ nmeth.2019 (2012).

42. Li, L. C. \& Dahiya, R. MethPrimer: designing primers for methylation PCRs. Bioinformatics 18, 1427-1431 (2002).

\section{Acknowledgements}

This study was supported by the Eugene Kennedy Shriver National Institute of Child Health and Human Development (award HD089053) and the National Institute of General Medical Sciences (award GM073646).

\section{Author Contributions}

R.B.C., D.C.F., A.Q., and J.G.B. designed research; R.B.C., D.C.F., A.Q., and J.B performed research; R.B.C. and J.G.B. analyzed data, and wrote the manuscript. All authors reviewed the manuscript.

Additional Information

Supplementary information accompanies this paper at https://doi.org/10.1038/s41598-019-45203-1.

Competing Interests: The authors declare no competing interests.

Publisher's note: Springer Nature remains neutral with regard to jurisdictional claims in published maps and institutional affiliations.

(c) (i) Open Access This article is licensed under a Creative Commons Attribution 4.0 International License, which permits use, sharing, adaptation, distribution and reproduction in any medium or format, as long as you give appropriate credit to the original author(s) and the source, provide a link to the Creative Commons license, and indicate if changes were made. The images or other third party material in this article are included in the article's Creative Commons license, unless indicated otherwise in a credit line to the material. If material is not included in the article's Creative Commons license and your intended use is not permitted by statutory regulation or exceeds the permitted use, you will need to obtain permission directly from the copyright holder. To view a copy of this license, visit http://creativecommons.org/licenses/by/4.0/.

(C) The Author(s) 2019 